\title{
The cleaved-Caspase-3 antibody is a marker of Caspase-9-like DRONC activity in Drosophila
}

\author{
Y Fan $^{1}$ and A Bergmann ${ }^{* 1}$
}

The cleaved-Caspase-3 antibody is a popular tool in apoptosis research in Drosophila. As the antibody was raised against cleaved human Caspase-3, it was assumed that it detects cleaved DRICE and DCP-1, Caspase-3-like effector caspases in Drosophila. However, as shown here, strong immunoreactivity persists in apoptotic models doubly mutant for drICE and dcp-1. In contrast, mutants of the apoptosome components DRONC (Caspase-9-like) and ARK (Apaf-1 related) do not label with the cleaved-Caspase-3 antibody. By peptide blocking experiments and further genetic studies, we provide evidence that the cleavedCaspase-3 antibody recognizes multiple proteins including DCP-1 and likely DRICE, but also at least one additional unknown protein, all of which require DRONC for epitope exposure. The unknown substrate may be involved in non-apoptotic functions of DRONC. Because the cleaved-Caspase-3 antibody not only detects cleaved Caspase-3-like proteins in Drosophila, but also other proteins in a DRONC-dependent manner, it is more accurate to consider the cleaved-Caspase-3 antibody as a marker for DRONC activity, rather than effector caspase activity.

Cell Death and Differentiation (2010) 17, 534-539; doi:10.1038/cdd.2009.185; published online 4 December 2009

The cleaved-Caspase-3 (Asp175) antibody (referred to as cleaved-Caspase-3 antibody) from Cell Signaling Technology (Danvas, MA, USA) is a polyclonal antibody obtained from rabbit that was raised against a peptide in the large subunit of the human effector caspase, Caspase-3, amino-terminal to Asp $175 .{ }^{1}$ The antibody does not detect unprocessed Caspase-3. However, after proteolytic cleavage between Asp175 and Ser176, separating the large and small subunits leads to the activation of Caspase-3, the epitope is exposed and can be detected by cleaved-Caspase- 3 antibody, thus making the antibody a marker for cleaved and active Caspase-3 in dying cells. A similar antibody, termed CM1, has previously been described; $; 2,3$ however, the CM1 antibody is no longer available and is not a subject of this analysis.

Apoptosis in Drosophila is under the control of pro-apoptotic genes reaper, hid and grim. ${ }^{4-6}$ (reviewed $\mathrm{in}^{7}$ ) The products of these genes trigger apoptosis through the induction of proteolytic degradation of inhibitor of apoptosis proteins (IAPs), most notably DIAP $1,{ }^{8-11}$ to activate a caspase program. Of the seven caspase genes in Drosophila, ${ }^{7}$ only the putative initiator caspase DRONC which is most similar to mammalian Caspase-9, and the Caspase-3-like effector caspases DRICE and DCP-1 have been implicated in developmental apoptosis. ${ }^{12-18}$ After its release from DIAP1 inhibition, DRONC becomes a part of the apoptosome through interaction with ARK, also known as HAC-1 and
D-APAF-1, the APAF-1-related gene in Drosophila. ${ }^{19-21}$ The apoptosome cleaves and activates the effector caspases DCP-1 and DRICE.

The cleaved-Caspase-3 antibody has become a very popular tool for detection of dying cells in Drosophila (see for example references ${ }^{3,13,22-27}$ ). As it has been raised against an epitope of human Caspase-3, it was proposed that the antibody would cross-react with the cleaved Caspase-3like effector caspases DRICE and DCP-1 in Drosophila. ${ }^{3}$ However, this has never been rigorously tested. Previously, it was shown that the antibody does not loose its immunoreactivity in $d r I C E$ single mutants, ${ }^{15}$ thus suggesting that it is not specific for cleaved DRICE. However, partial redundancy with DCP-1 (ref. ${ }^{13}$ ) may account for this result. Here, we show that the cleaved-Caspase-3 antibody still labels cells induced to die, which are double mutants for defined null alleles of $d c p-1$ and $d r I C E$. In contrast, the antibody requires the activity of the apoptosome components DRONC and ARK for immunoreactivity. Subsequent analysis demonstrates that the cleaved-Caspase-3 antibody detects at least one additional putative DRONC substrate that may be involved in non-apoptotic processes. Because the cleaved-Caspase-3 antibody is not entirely specific for cleaved DRICE and DCP-1, but requires DRONC for its immunoreactivity, we propose that it is more accurate to refer to this antibody as a marker of DRONC activity in Drosophila.

\footnotetext{
${ }^{1}$ Department of Biochemistry and Molecular Biology, Graduate Program in Genes and Development, The University of Texas MD Anderson Cancer Center, 1515 Holcombe Boulevard-Unit 1000, Houston, TX 77030, USA

${ }^{*}$ Corresponding author: A Bergmann, Department of Biochemistry and Molecular Biology, Graduate Program in Genes and Development, MD Anderson Cancer Center, 1515 Holcombe Boulevard-Unit 1000, Houston, TX 77030, USA. Tel: + 1713834 6294; Fax: + 1713834 6291; E-mail: abergman@mdanderson.org Keywords: cleaved-Caspase-3; Drosophila; DRICE; DRONC; Dcp-1

Abbreviations: APAF-1, apoptosis peptidase-activating protein-1; ARK, apoptosis peptidase-activating protein-1-related killer; D-APAF-1, Drosophila apoptosis peptidase-activating protein-1; DCP-1, death caspase-1; $\Delta \mathrm{N}$, delta N-terminus; DIAP-1, Drosophila inhibitor of apoptosis protein-1; DRICE, Drosophila interleukinconverting enzyme; DRONC, Drosophila Nedd2-like caspase; ETD, Glu-Thr-Asp; GMR, glass multimer reporter; HAC-1, homolog of APAF-1 and Ced4-1; IAP, inhibitor of apoptosis protein; TUNEL, terminal deoxynucleotidyl transferase mediated dUTP-nick end labeling

Received 22.7.09; revised 02.11.09; accepted 02.11.09; Edited by RA Knight; published online 04.12.09
} 


\section{Results}

Labeling by the cleaved-Caspase-3 antibody persists in dcp-1 drICE double mutants. To evaluate the specificity of the cleaved-Caspase- 3 antibody, we analyzed eye imaginal discs from third instar larvae. In wild-type eye imaginal discs, the antibody detects a few dying cells scattered throughout the disc (Figure 1a). Surprisingly, in eye discs doubly mutant for the null alleles $d c p-1^{\text {Prev }}$ and $d r l C E^{\Delta 1}$ (ref. ${ }^{14,15}$ ), the cleaved-Caspase-3 antibody still labels cells (Figure 1b). Labeling in $d c p-1^{\text {Prev }} d r l C E^{\Delta 1}$ double mutants occurs in clusters (Figure 1b), similar to what has been observed previously when cell death was blocked by the expression of Caspase-3 inhibitor P35. ${ }^{3}$ These observations would suggest that the cleaved-Caspase- 3 antibody still detects an epitope in the absence of the Caspase-3-like proteins DCP-1 and DRICE. Nevertheless, as apoptosis at this stage does not occur in a defined pattern, we were uncertain about the specificity of these labeling signals.

Therefore, we used GMR-hid transgenes, a well characterized apoptotic model, ${ }^{5}$ to further examine the specificity of the cleaved-Caspase-3 antibody. Through GMR-driven expression of the pro-apoptotic gene hid specifically in the posterior half of the developing eye, GMR-hid transgenes induce apoptosis in two distinct waves as shown by cleavedCaspase-3 antibody and TUNEL labeling ${ }^{28}$ (Figure 1c,d). To evaluate the specificity of the cleaved-Caspase-3 antibody, we examined GMR-hid eye discs that were doubly mutant for $d c p-1^{\text {Prev }}$ and $d r l C E^{41}$. Consistent with the expectation, loss of $d c p-1^{\text {Prev }}$ and $d r l C E^{\Delta 1}$ completely abrogates TUNELpositive apoptosis in GMR-hid discs (Figure 1f). Surprisingly, however, $d c p-1^{\text {Prev }} d r l C E^{41}$ double mutant GMR-hid eye discs still showed strong immunoreactivity with cleavedCaspase-3 antibody (Figure 1e). Thus, the cleaved-Caspase-3 antibody does not or not only detect DRICE and/or DCP-1. We do note, though, that the labeling appearance of the cleaved-Caspase-3 antibody changes in the absence of DCP-1 and DRICE (compare Figure 1c and e). The labeling signal is no longer restricted to two distinct waves (Figure 1c), but rather fills the entire posterior compartment of the eye disc and is confined to interommatidial cells (Figure 1e). A similar change of labeling pattern has been reported for CM1 antibody labeling upon expression of the caspase inhibitor P35. ${ }^{3}$ This change of the labeling pattern is likely because of the fact that cells in $d c p-1^{\text {Prev }} d r I C E^{\Delta 1}$ double mutant GMR-hid eye discs do not die (Figure 1f) and thus, maintain the epitope detected by cleaved-Caspase-3 antibody. However, it is important to note that this analysis demonstrates the detection of an epitope in the absence of Caspase-3-like proteins DCP-1 and DRICE by cleaved-Caspase-3 antibody.

Immunoreactivity of the cleaved-Caspase-3 antibody depends on the apoptosome components DRONC and ARK. There are two possibilities why the cleavedCaspase-3 antibody labels $d c p-1 d r l C E$ double mutant cells,
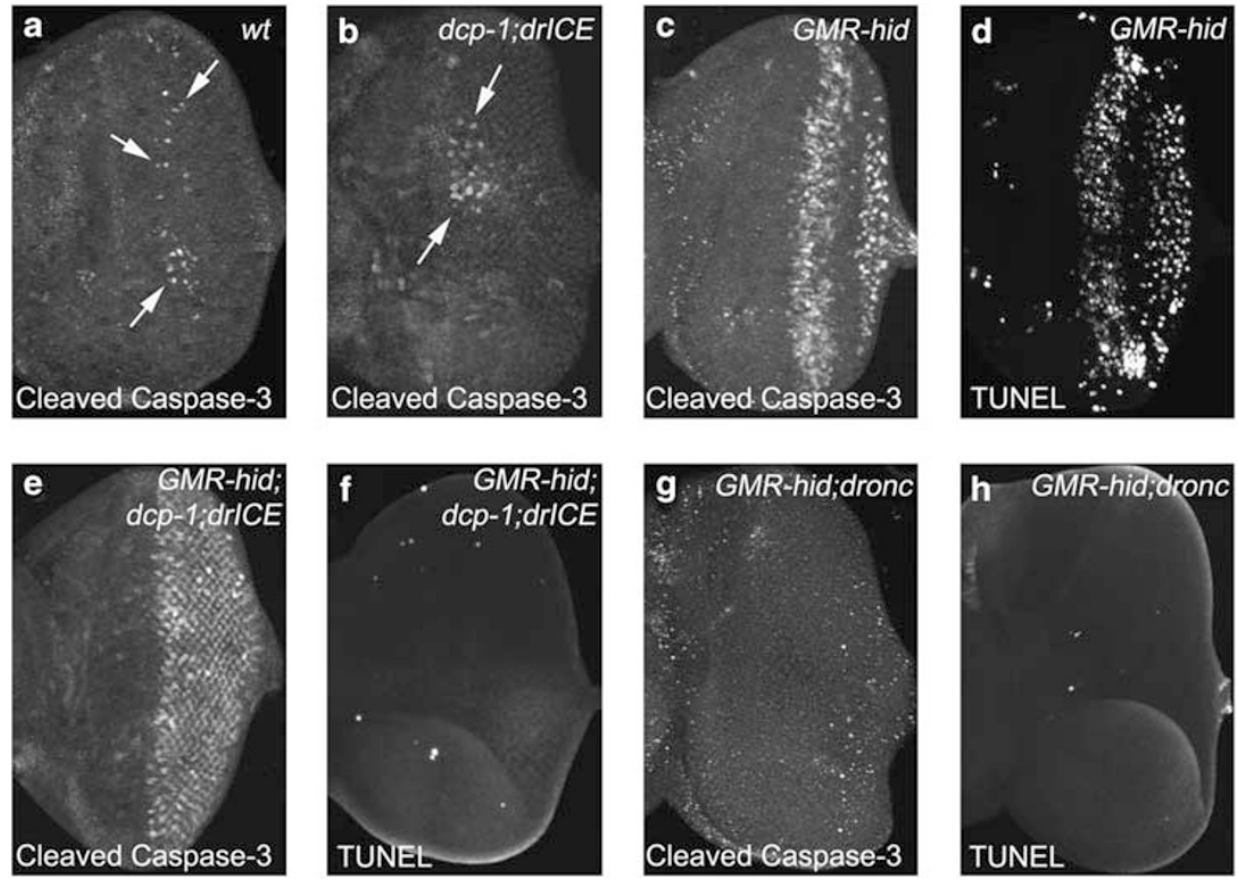

Figure 1 The cleaved-Caspase-3 antibody is a marker for DRONC activity. Shown are eye imaginal discs of third instar larvae. Posterior is to the right. GMR-hid is a transgenic insertion on the $\mathrm{X}$ chromosome.(a) Wild-type (wt) disc labeled with cleaved-Caspase-3 antibody. Arrows point to a few immunopositive cells.(b) A disc doubly mutant for the null alleles $d c p-1^{P r e v}$ and $d r l C E^{\Delta 1}$ labeled with cleaved-Caspase-3 antibody. Arrows point to a few immunopositive cells.(c) and (d) GMR-hid eye discs in otherwise wild-type background labeled with (c) cleaved-Caspase-3 antibody and (d) TUNEL. Note the strong signals in the posterior half of the eye discs. (e) and (f) GMR-hid eye discs doubly mutant for $d c p-1^{\text {Prev }}$ and $d r I C E^{\Delta 1}$ labeled for (e) cleaved-Caspase-3 antibody and (f) TUNEL. Although TUNEL labeling is completely blocked, cleaved-Caspase-3 antibody still delivers a strong signal in the posterior half of the eye disc. (g) and (h) GMR-hid eye discs mutant for the null allele dronc ${ }^{124}$. Both $(\mathbf{g})$ cleavedCaspase-3 antibody and (h) TUNEL labeling are blocked by loss of DRONC. Genotypes: (a) wild-type; (b) dcp-1 Prev $/ d c p-1^{\text {Prev }}$; drICE $E^{\Delta 1} / d r I C E^{\Delta 1}$; (c) and (d) GMR-hid/GMR-

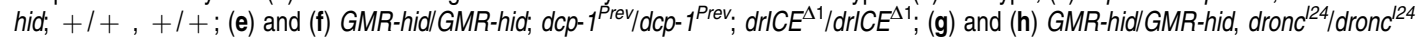


although they are not apoptotic. First, the antibody may not detect an apoptotic epitope; or second, the antibody may detect an apoptotic epitope generated upstream or in parallel to DCP-1 and DRICE. To distinguish between these possibilities, we examined GMR-hid eye discs mutant for the apoptosome components DRONC and ARK, which act upstream of DRICE and DCP-1. In dronc and ark mutant GMR-hid eye discs, both TUNEL and cleaved-Caspase-3 antibody labelings are blocked (Figure $1 \mathrm{~g}, \mathrm{~h}$; Figure 3a,b). These data confirm that the cleaved-Caspase-3 antibody indeed detects an apoptotic epitope in Drosophila. Furthermore, because it fails to detect the apoptotic epitope in dronc and ark mutants, but not in dcp-1 drICE double mutants, it is more accurate to consider the cleavedCaspase-3 antibody as a marker for DRONC activity, rather than effector caspase activity, in dying Drosophila cells.

The tripeptide ETD is the apoptotic epitope detected by cleaved-Caspase-3 antibody. The epitope detected by the cleaved-Caspase-3 antibody depends on DRONC activity. It may be possible that the antibody directly recognizes activated DRONC. Alternatively, it is also possible that the cleaved-Caspase-3 antibody detects an epitope generated through the cleavage of an unknown substrate by active DRONC, independently of DRICE and DCP-1.

To distinguish between these two possibilities, we aligned the residues from the catalytic cysteine (Cys163) to the cleavage site at Asp175 of human Caspase-3 (Caspase-3 peptide) with the corresponding regions of the Drosophila caspases (Figure $2 \mathrm{a}$; see also ref. ${ }^{3}$ ). The most C-terminal residues of the Caspase-3 peptide, ETD, are conserved in DRICE and DCP-1 (Figure 2a). Similar to Caspase-3, this is the cleavage site for activation of at least DRICE, ${ }^{29}$ and possibly DCP-1. It is interesting to note that the $\mathrm{N}$-terminal, two-third of the Caspase-3 peptide, bears highest similarity to DRONC; six out of nine residues are conserved (Figure 2a). This part of the Caspase-3 peptide is less well-conserved in DRICE, DCP-1 and the remaining Drosophila caspases. Although cleavage between the large and small subunits of DRONC is not necessary for its activity, $^{30,31}$ and may not occur in vivo, we considered the possibility that antibodies directed against the $\mathrm{N}$-terminal part of Caspase-3 peptide may directly detect active DRONC in dying cells.

We used blocking peptides to evaluate which epitopes of the Caspase-3 peptide are detected by the cleavedCaspase-3 antibody in dying Drosophila cells. The sequences of the blocking peptides are shown in Figure $2 \mathrm{~b}$ and underlined in Figure 2a. Blocking peptide $A$ (TETD) is derived from DRICE and DCP-1 and blocking peptide $B$ (CRGDEYDLG) corresponds to the region of highest similarity in DRONC (Figure 2a,b). Peptide $\mathrm{C}$ is a control peptide corresponding to the residues immediately adjacent to peptide $B$ in DRONC (Figure 2a,b). Peptide D is another control peptide, which is very similar to peptide $A$ and is derived from the prodomain of DRONC at position 113. If the prodomain of DRONC is cleaved at this site, it will expose ESD at its C-terminus, which is very similar to the C-terminus of the Caspase-3 peptide (ETD, peptide A) and may, thus, be detected by the cleaved-Caspase-3 antibody.

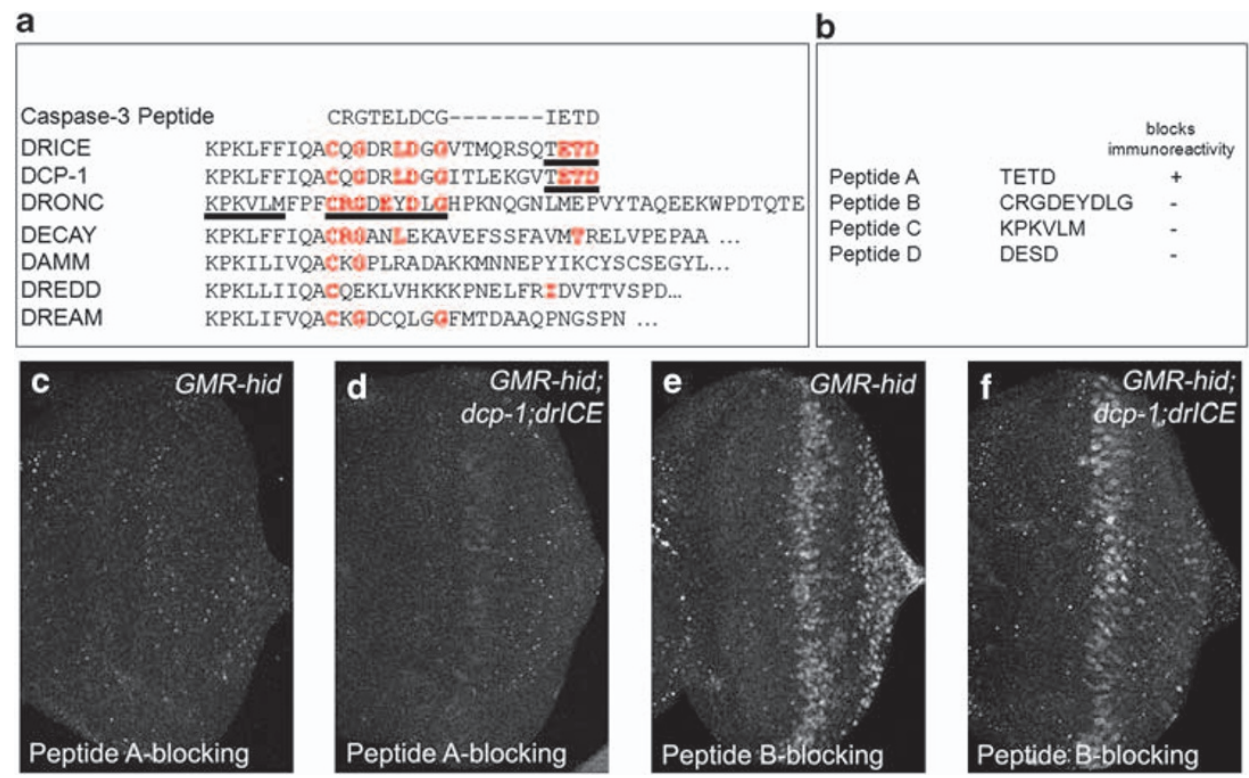

Figure 2 A peptide containing ETD blocks cleaved-Caspase-3 immunoreactivity.(a) Amino-acid sequence alignment of the residues from catalytic Cys 163 to the cleavage site at Asp175 of human Caspase-3 (Caspase-3 peptide) and the corresponding regions of the Drosophila caspases. Residues highlighted in red are identical in the Caspase3 peptide. For DRICE, DCP-1 and DRONC cleavage has been demonstrated following the last residue ( $\mathbf{d}$ and $\mathbf{e})$ of the sequence shown. For DECAY, DAMM, DREDD and DREAM cleavage is uncertain and the end of the sequence shown does not imply cleavage (indicated by ...). Underlined sequences were used in blocking peptides (compare with b). (b) Amino-acid sequences of the blocking peptides. Only peptide A blocks immunoreactivity of the cleaved-Caspase-3 antibody. (c and d) Preincubation of cleavedCaspase-3 antibody with peptide A completely abrogates its immunoreactivity in GMR-hid eye discs (c) and GMR-hid eye discs mutant for $d c p-1$ and $d r l C E$ (d). (e and f) Preincubation of cleaved-Caspase-3 antibody with peptide B has little or no effect on its immunoreactivity in GMR-hid eye discs (e) and GMR-hid eye discs mutant for dcp-1 and $\operatorname{drlCE}(\mathbf{f})$. Similar results were obtained for peptides $\mathbf{c}$ and $\mathbf{d}$ (data not shown) 

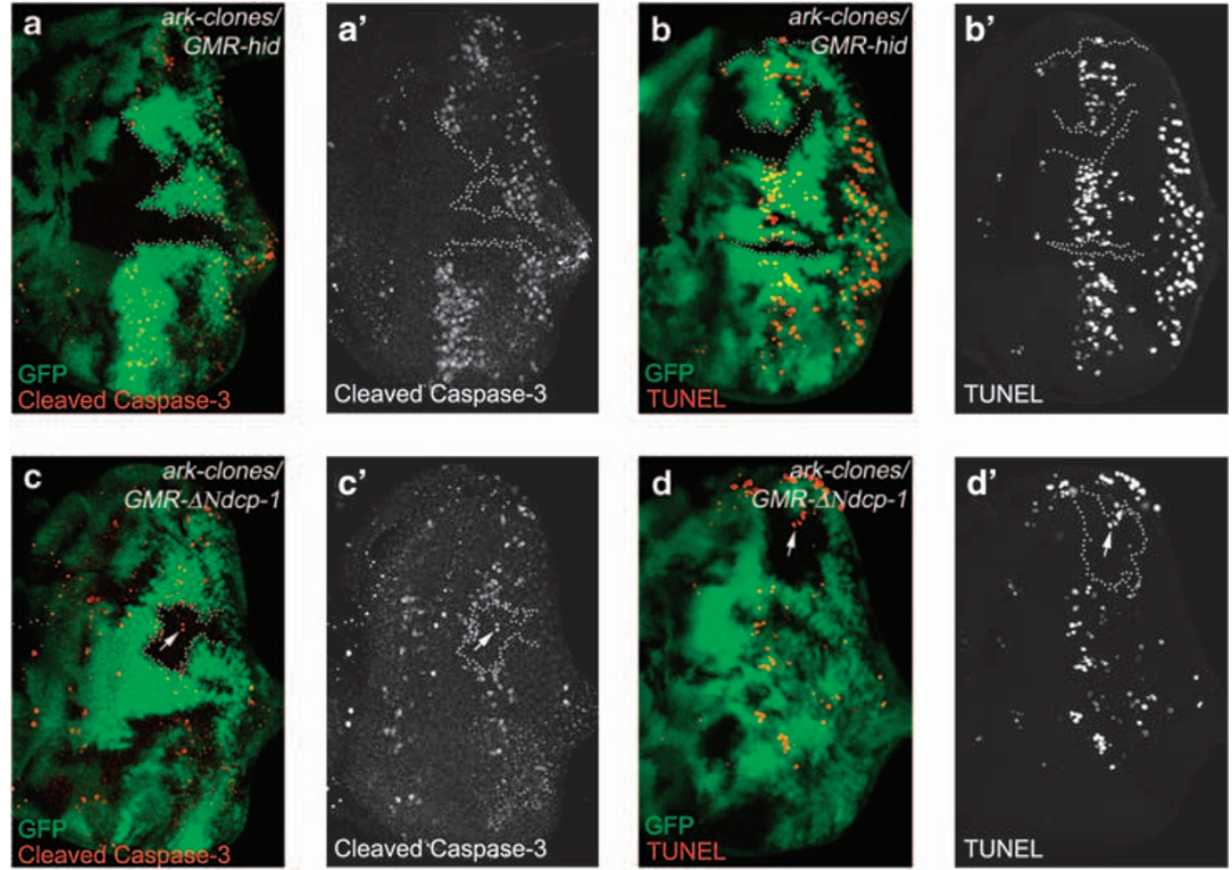

Figure 3 Expression of a $\Delta N-d c p-1$ transgene in ark mutant clones partially restores cleaved-Caspase-3 immunoreactivity. (a, a', b, b') GMR-hid eye discs containing ark mutant clones were labeled with cleaved-Caspase-3 antibody (a, $\left.\mathbf{a}^{\prime}\right)$ and TUNEL (b, $\left.\mathbf{b}^{\prime}\right)$. ark mutant clones are marked by absence of GFP. A few clonal boundaries are indicated by stippled lines. Both the cleaved-Caspase-3 and TUNEL signals are lost in ark clones. (a') and (b') are the cleaved-Caspase-3 and TUNEL channels only. Genotype: GMR-hid ey-FLP; FRT42D ark ${ }^{G 8} / F R T 42 D$ P(ubi-GFP). (c, c', d, d') GMR- $\triangle N$-dcp-1 eye discs containing ark mutant clones were labeled with cleaved-Caspase-3 antibody (c, $\mathbf{c}^{\prime}$ ) and TUNEL (d, d'). ark mutant clones are marked by the absence of GFP. In ark ${ }^{+}$tissue, marked by GFP (green), cleaved-Caspase-3 and TUNEL signals are easily detectable. In ark mutant clones (see outline of clonal boundaries by stippled lines), the number of both cleaved-Caspase-3- and TUNEL-positive cells is reduced, but a few are present (arrows). (c') and (d') are the cleaved-Caspase-3 and TUNEL channels only. Genotype: ey-FLP; FRT42D ark ${ }^{\mathrm{G} 8} /$ /FRT42D P(ubi-GFP); GMR- $\triangle N-d c p-1$

The blocking peptides were mixed with the cleavedCaspase-3 antibody 60 min before incubation with the eye imaginal discs. The results of the blocking experiments are summarized in Figure $2 b$, and for peptides $A$ and $B$ shown in Figure 2c-f. Peptide $A$ is sufficient to block the entire immunoreactivity of the cleaved-Caspase-3 antibody in GMR-hid and in dcp-1 drICE double mutant GMR-hid eye discs (Figure 2c,d). In contrast, peptide B does not abrogate immunoreactivity of the antibody in these discs (Figure 2e,f). The control peptides $C$ and $D$ also fail to block cleavedCaspase-3 immunoreactivity (Figure $2 \mathrm{~b}$; data not shown).

These data demonstrate that the cleaved-Caspase-3 antibody specifically detects the epitope ETD in apoptotic cells. Among the Drosophila caspases, this epitope is only present in DRICE and DCP-1, therefore making it very likely that the antibody does indeed detect these effector caspases. In contrast, the fact that the DRONC-derived peptides $\mathrm{B}, \mathrm{C}$ and $D$ fail to block immunoreactivity suggest that it is unlikely for the antibody to directly detect active DRONC. Therefore, because the cleaved-Caspase- 3 antibody does not lose immunoreactivity in $d c p-1 d r l C E$ double mutants (Figure 1e), it detects at least one other protein, which exposes the ETD epitope in a DRONC-dependent manner.

Activation of DCP-1 independently of ARK restores cleaved-Caspase-3 immunoreactivity. The analysis presented in Figure 2 suggests, but does not prove, that the cleaved-Caspase-3 antibody does indeed detect cleaved
DCP-1 and DRICE. To directly test this possibility, we expressed a GMR- $\Delta N-d c p-1$ transgene in ark mutant background. $\Delta N-d c p-1$ lacks the $\mathrm{N}$-terminal prodomain of DCP-1. It is thought that prodomain-depleted $\triangle \mathrm{N}-\mathrm{DCP}-1$ readily promotes autoprocessing, and consistent expression under GMR control induces an eye ablation phenotype. ${ }^{32}$ This eye ablation phenotype is caused by the induction of apoptosis (Figure 3c,d). As mentioned above, ark mutant clones in GMR-hid background fail to induce TUNEL-positive apoptosis (Figure $3 \mathrm{~b}$ ) and the cleaved-Caspase-3 antibody does not have any immunoreactivity in ark clones (Figure 3a) suggesting that neither DCP-1 nor DRICE nor the unknown DRONC substrates are cleaved in ark mutant clones. Therefore, we tested whether expression of $\Delta \mathrm{N}-D c p-1$ in the absence of apoptosome activity, that is, in ark clones, can restore cleaved-Caspase-3 antibody labeling. Compared with wild-type tissue (marked by GFP in Figure $3 c, c^{\prime}$ ), the number of cleaved-Caspase-3 positive cells is strongly reduced in ark mutant clones (Figure 3c,c') suggesting that activation of $\triangle \mathrm{N}-\mathrm{DCP}-1$ is at least partially dependent on the apoptosome. However, about $50 \%$ of ark mutant clones $(n=32)$ in GMR- $\Delta N-D c p-1$ background contain cleavedCaspase-3 immunoreactive cells (arrows in Figure 3c, c'). This is unlikely to be a labeling artifact, because these cells also are TUNEL-positive (Figure 3d, d'). Therefore, because DRONC is inactive in ark mutant background suggesting that the cleaved-Caspase-3 labeling signal is not caused by the unknown DRONC substrate, this analysis demonstrates that 


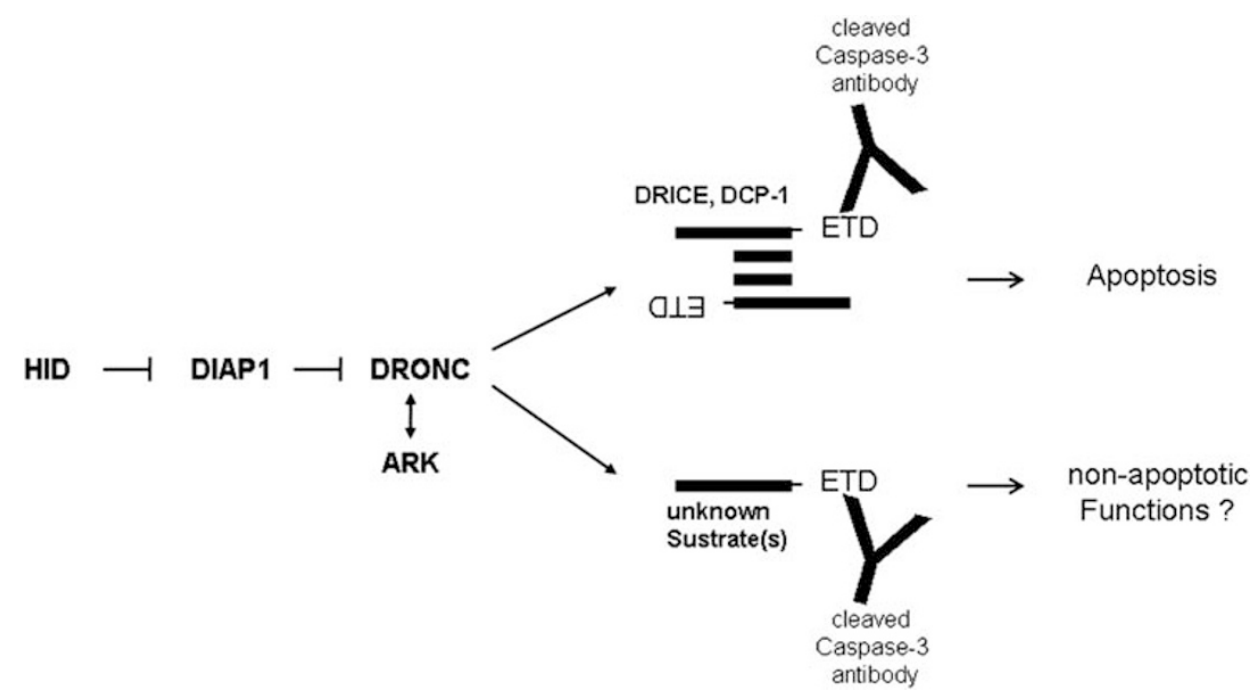

Figure 4 Specificity of the cleaved-Caspase-3 antibody. HID-mediated release of DRONC from DIAP1-inhibition triggers the formation of apoptosome through association with ARK. After DRONC cleavage, the apoptotic substrates DRICE and DCP-1 form a heterotetramer, exposing the ETD epitope at the C-terminus of the large subunits of these proteins. In addition, DRONC has at least one additional substrate, which likely exposes an ETD epitope after cleavage. This substrate is unknown and may mediate non-apoptotic functions of DRONC. The cleaved-Caspase-3 antibody (Y shape) detects the exposed ETD epitope of cleaved DCP-1, DRICE and the unknown DRONC substrate

the cleaved-Caspase-3 antibody does indeed detect cleaved DCP-1. It is also possible that the cleaved-Caspase-3 antibody detects cleaved DRICE in this experiment, because DCP-1 can proteolytically process DRICE, at least in vitro. ${ }^{29,32} \mathrm{~A}$ similar analysis with DRICE could not be carried out because GMR-drICE and GMR- $\triangle N-d r I C E$ do not cause an eye ablation phenotype. ${ }^{32}$ Nevertheless, whether DCP-1 cleaves DRICE in vivo or not, given the sequence similarity of DCP-1 and DRICE at the C-terminus of the large subunit and the peptide blocking data of Figure 2, it suggests that the antibody may also detect cleaved DRICE.

\section{Discussion}

The findings of this study are summarized in Figure 4 . In addition to DCP-1 and likely DRICE, the cleaved-Caspase-3 antibody also recognizes at least one, perhaps even several additional proteins containing the ETD epitope. Because exposure of this epitope is dependent on apoptosome activity, it is very likely that the protein is a substrate of DRONC. However, cleavage of this protein by DRONC is not sufficient to induce apoptosis because $d c p-1 \mathrm{dr} / C E$ double mutants in which this epitope is present, are not apoptotic (Figure 1e,f). Consistently, this protein is unlikely to be a caspase because none of the remaining Drosophila caspases carries the ETD epitope (Figure 2a).

Nevertheless, these findings are interesting from a different point of view. In addition to induction of apoptosis, DRONC and caspases in general also have non-apoptotic functions (reviewed in ${ }^{33}$ ). For example, apoptosis-induced compensatory proliferation is dependent on a non-apoptotic function of DRONC. ${ }^{16,34,35}$ It is at present unknown how DRONC is mediating this response. Identification of DRONC substrates involved in non-apoptotic functions may be very helpful in elucidating the mechanism by which DRONC exerts its non-apoptotic functions. Unfortunately, database searches using ETD as query to identify potential non-apoptotic DRONC substrates were not successful. Other approaches such as immunoprecipitation using the cleaved-Caspase-3 antibody will be necessary to identify this substrate.

This study also demonstrates that caution should be taken about the specificity of antibodies. As shown here in Drosophila, it is likely that the cleaved-Caspase-3 antibody not only detects cleaved Caspase- 3 in mammals, but also additional proteins containing the ETD epitope. In Drosophila, exposure of this epitope is apoptosome-dependent and thus, the antibody is a useful reagent for detecting apoptosis. But in other model organisms this may not be the case and may result in mis-interpretations.

In summary, we demonstrated in this paper that the cleaved-Caspase-3 antibody not only detects active effector caspases DRICE and DCP-1 in apoptotic cells in Drosophila, but also additional potential DRONC substrates which may be involved in non-apoptotic processes. Therefore, we propose that it is most useful to interpret the labeling information obtained with the cleaved-Caspase- 3 antibody as the activity of Caspase-9-like initiator caspase DRONC in Drosophila.

\section{Materials and Methods}

The cleaved-Caspase-3 (Asp175) antibody was purchased from Cell Signaling Technology (catalog \# 9661). 1 GMR-hid, GMR- $\Delta N-d c p-1$, dronc ${ }^{124}$, ark ${ }^{G 8}$, drICE ${ }^{\Delta 1}$ and $d c p-1^{\text {Prev }}$ are described elsewhere. ${ }^{5,12,14,15,32,36}$ For details about making ark clones, see Srivastava et al. $\left(\right.$ ref. $\left.{ }^{36}\right)$. Immunohistochemistry and TUNEL labeling was done as described. ${ }^{22,28,37}$ Photographs were taken by confocal microscopy.

Blocking peptides A, B, C, and D were obtained from Prolmmune (Oxford, UK). They were dissolved in a small amount of $0.1 \%$ ammonium bicarbonate and diluted to a final concentration of $1 \mathrm{mg} / \mathrm{ml}$ with sterile, distilled water according to the manufacturer's instructions. $1 \mu \mathrm{l}$ of the peptide solution was mixed with $1 \mu \mathrm{l}$ of cleaved-Caspase-3 antibody in labeling solution for $60 \mathrm{~min}$ at room temperature before incubation with the eye discs. 


\section{Conflict of interest}

The authors declare no conflict of interest.

Acknowledgements. We thank Kim McCall, Hermann Steller and Bruce Hay for fly stocks. This work was supported by the NIH (GM068016, GM081543, GM074977) and The Welch Foundation (G-1496).

1. See Cell Signaling Technology website. http://www.cellsignal.com/pdt/9661.pdf.

2. Srinivasan A, Roth KA, Sayers RO, Shindler KS, Wong AM, Fritz LC et al. In situ immunodetection of activated caspase-3 in apoptotic neurons in the developing nervous system. Cell Death Differ 1998; 5: 1004-1016.

3. Yu SY, Yoo SJ, Yang L, Zapata C, Srinivasan A, Hay BA et al. A pathway of signals regulating effector and initiator caspases in the developing Drosophila eye. Development 2002; 129: 3269-3278

4. White K, Grether ME, Abrams JM, Young L, Farrell K, Steller H. Genetic control of programmed cell death in Drosophila. Science 1994; 264: 677-683.

5. Grether ME, Abrams JM, Agapite J, White K, Steller H. The head involution defective gene of Drosophila melanogaster functions in programmed cell death. Genes Dev 1995; 9 1694-1708.

6. Chen P, Nordstrom W, Gish B, Abrams JM. Grim, a novel cell death gene in Drosophila Genes Dev 1996; 10: 1773-1782.

7. Xu D, Woodfield SE, Lee TV, Fan Y, Antonio C, Bergmann A. Genetic control of programmed cell death (apoptosis) in Drosophila. Fly (Austin) 2009; 3: 78-90.

8. Ryoo HD, Bergmann A, Gonen H, Ciechanover A, Steller H. Regulation of Drosophila IAP1 degradation and apoptosis by reaper and ubcD1. Nat Cell Biol 2002; 4: 432-438.

9. Holley CL, Olson MR, Colon-Ramos DA, Kornbluth S. Reaper eliminates IAP proteins through stimulated IAP degradation and generalized translational inhibition. Nat Cell Biol 2002; 4: 439-444.

10. Hays R, Wickline L, Cagan R. Morgue mediates apoptosis in the Drosophila melanogaster retina by promoting degradation of DIAP1. Nat Cell Biol 2002; 4: 425-431.

11. Yoo SJ, Huh JR, Muro I, Yu H, Wang L, Wang SL et al. Hid, Rpr and Grim negatively regulate DIAP1 levels through distinct mechanisms. Nat Cell Biol 2002; 4: 416-424.

12. Xu D, Li Y, Arcaro M, Lackey M, Bergmann A. The CARD-carrying caspase Dronc is essential for most, but not all, developmental cell death in Drosophila. Development 2005; 132: 2125-2134.

13. Xu D, Wang Y, Willecke R, Chen Z, Ding T, Bergmann A. The effector caspases drICE and dcp-1 have partially overlapping functions in the apoptotic pathway in Drosophila. Cell Death Differ 2006; 13: 1697-1706.

14. Laundrie B, Peterson JS, Baum JS, Chang JC, Fileppo D, Thompson SR et al. Germline cell death is inhibited by P-element insertions disrupting the dcp-1/pita nested gene pair in Drosophila. Genetics 2003; 165: 1881-1888.

15. Muro I, Berry DL, Huh JR, Chen CH, Huang H, Yoo SJ et al. The Drosophila caspase Ice is important for many apoptotic cell deaths and for spermatid individualization, a nonapoptotic process. Development 2006; 133: 3305-3315.

16. Kondo S, Senoo-Matsuda N, Hiromi Y, Miura M. DRONC coordinates cell death and compensatory proliferation. Mol Cell Biol 2006; 26: 7258-7268.

17. Chew SK, Akdemir F, Chen P, Lu WJ, Mills K, Daish T et al. The apical caspase Dronc governs programmed and unprogrammed cell death in Drosophila. Dev Cell 2004; 7 897-907.
18. Daish TJ, Mills K, Kumar S. Drosophila caspase DRONC is required for specific developmental cell death pathways and stress-induced apoptosis. Dev Cell 2004; 7: 909-915.

19. Kanuka H, Sawamoto K, Inohara N, Matsuno K, Okano H, Miura M. Control of the cell death pathway by Dapaf-1, a Drosophila Apaf-1/CED-4-related caspase activator. Mol Cell 1999; 4: 757-769.

20. Rodriguez A, Oliver H, Zou H, Chen P, Wang X, Abrams JM. Dark is a Drosophila homologue of Apaf-1/CED-4 and functions in an evolutionarily conserved death pathway. Nat Cell Biol 1999; 1: 272-279.

21. Zhou L, Song Z, Tittel J, Steller H. HAC-1, a Drosophila homolog of APAF-1 and CED-4 functions in developmental and radiation-induced apoptosis. Mol Cell 1999; 4 : 745-755

22. Fan Y, Bergmann A. Distinct mechanisms of apoptosis-induced compensatory proliferation in proliferating and differentiating tissues in the Drosophila eye. Dev Cell 2008; 14: $399-410$.

23. Shapiro PJ, Hsu HH, Jung H, Robbins ES, Ryoo HD. Regulation of the Drosophila apoptosome through feedback inhibition. Nat Cell Biol 2008; 10: 1440-1446.

24. Peterson JS, Barkett M, McCall K. Stage-specific regulation of caspase activity in Drosophila oogenesis. Dev Biol 2003; 260: 113-123.

25. Arama E, Bader M, Srivastava M, Bergmann A, Steller H. The two Drosophila cytochrome $\mathrm{C}$ proteins can function in both respiration and caspase activation. EMBO J 2006; 25: 232-243.

26. Baum JS, Arama E, Steller H, McCall K. The Drosophila caspases Strica and Dronc function redundantly in programmed cell death during oogenesis. Cell Death Differ 2007; 14: 1508-1517.

27. Reis $T$, Edgar BA. Negative regulation of $d E 2 F 1$ by cyclin-dependent kinases controls cell cycle timing. Cell 2004; 117: 253-264.

28. McCall K, Peterson JS. Detection of apoptosis in Drosophila. Methods Mol Biol 2004; 282: 191-205.

29. Hawkins CJ, Yoo SJ, Peterson EP, Wang SL, Vernooy SY, Hay BA. The Drosophila caspase DRONC cleaves following glutamate or aspartate and is regulated by DIAP1, HID, and GRIM. J Biol Chem 2000; 275: 27084-27093.

30. Dorstyn L, Kumar S. A biochemical analysis of the activation of the Drosophila caspase DRONC. Cell Death Differ 2008; 15: 461-470.

31. Snipas SJ, Drag M, Stennicke HR, Salvesen GS. Activation mechanism and substrate specificity of the Drosophila initiator caspase DRONC. Cell Death Differ 2008; 15: $938-945$.

32. Song Z, Guan B, Bergman A, Nicholson DW, Thornberry NA, Peterson EP et al. Biochemical and genetic interactions between Drosophila caspases and the proapoptotic genes rpr, hid, and grim. Mol Cell Biol 2000; 20: 2907-2914.

33. Kuranaga E, Miura M. Nonapoptotic functions of caspases: caspases as regulatory molecules for immunity and cell-fate determination. Trends Cell Biol 2007; 17: 135-144.

34. Huh JR, Guo M, Hay BA. Compensatory proliferation induced by cell death in the Drosophila wing disc requires activity of the apical cell death caspase Dronc in a nonapoptotic role. Curr Biol 2004; 14: 1262-1266.

35. Fan Y, Bergmann A. The cell is dead. Long live the cell!. Trends Cell Biol 2008; 18: 467-473

36. Srivastava M, Scherr H, Lackey M, Xu D, Chen Z, Lu J et al. ARK, the Apaf-1 related killer in Drosophila, requires diverse domains for its apoptotic activity. Cell Death Differ 2007; 14: 92-102.

37. Arama E, Steller H. Detection of apoptosis by terminal deoxynucleotidyl transferasemediated dUTP nick-end labeling and acridine orange in Drosophila embryos and adult male gonads. Nat Protoc 2006; 1: 1725-1731. 